\title{
PREDICTION AND MEASUREMENT OF MICROSEGREGATION AND MICROSTRUCTURAL EVOLUTION IN DIRECTIONALLY SOLIDIFIED SUPERALLOYS
}

\author{
B. Böttger, U. Grafe, D. Ma \\ ACCESS e. V., Intzestraße 5, D-52056 Aachen, Germany \\ A. Schnell \\ ABB Management AG, Research Center CH-5405 Baden-Dättwil, Switzerland
}

\begin{abstract}
$\underline{\text { Abstract }}$
Microsegregation and microstructural evolution in directionally solidified superalloys is calculated using a unit cell approach. The results are comparcd to experimental micrographs and composition profiles. CMSX-4 and Inconel 706 are used as examples for a single-crystal and a conventional superalloy respectively. Two micromodels are applied to simulate solidification of the two alloys. For CMSX-4 as a typical SX alloy the SX-Model is formulated based on the Scheil approximation. For conventional superalloys as IN706 however carbon as interstitial element leads to considerable solid-state back diffusion. For that reason the more sophisticated Shape-Constrained Phasefield Model (SCPF-Model) is presented, which applies for diffusion in all phases and includes precipitation of secondary phases. In both models thermodynamic databases assessed using the CALPHAD method are coupled using the ThermoCalc software package.
\end{abstract}

\section{Introduction}

Solidification of muiticomponent alloys such as $\mathrm{Ni}$-base superalloys under practical conditions is always associated with solute redistribution of the alloying elements. Therefore formation of chemical inhomogenities and the consequent precipitation of interdendritic phases can be observed. Such inhomogeneous microstructures result in poor mechanical properties, even after heat treatment. For that reason it is required to predict segregation and secondary phase precipitation for different process parameters as well as for different alloy compositions.

Extensive work has been done to predict microsegregetion in dendritic microstructures. Frequently one-dimensional plate morphologies are used to describe the growing of secondary dendrite $\operatorname{arms}^{1-4}$. The half dendritic secondary arm spacing $\lambda_{2} / 2$ is used as length of the unit cell. Some models include coarsening of secondary arms ${ }^{3.4}$.

In processes like electroslag remelting (ESR) or vacuum arc remelting (VAR) dendritic growth of $\mathrm{Ni}$-base superalloys normally is near to the cellular-dendritic transition region due to the high thermal gradients. No tertiary arms are observed and the dendrites form a more or less regular array. In such cases one-dimensional cylindrical models have been formulated, which use the primary dendritic distance $\lambda_{1}$ as the dimension for the unit cell ${ }^{5,6}$. Recently $\mathrm{Ma}^{7}$ proposed a pseudo-2D model for cellular dendritic solidification of $\mathrm{Ni}$-base superalloys. This approach uses a fourfold shape function for description of the isothermal cross--section perpendicular to the growth direction of the dendrite. 
Superalloys are complex multicomponent systems. Generally the correct thermodynamic description is much more important than sophisticated kinetic modelling to get results, which are comparable to experimental findings ${ }^{8}$. Multicomponent systems are often described by linearized or interpolated phase diagrams ${ }^{4.10}$. This is a crude approximation, especially because tielines are not specified. Thermodynamic databases instead provide Gibbs energy phase descriptions of the system, which are based on a large number of experiments and are assessed by the CALPHAD 11 approach. By total Gibbs energy minimization phase equilibria can be obtained using software packages such as Thermo-Calc ${ }^{12}$. In the case of superalloys such databases have been developed including up to 13 elements and a large number of phases of practical interest ${ }^{13}$ as well.

\section{The unit cell approach}

The two models used here are based on a unit cell approach for directional dendritic solidification which makes use of a fourfold shape function for the approximation of the isothermal cross section of a dendrite, like that shown in Fig. 1, and was proposed by $\mathrm{Ma}^{7}$ :

$$
L(\beta)=L_{0}(t)(1+A \cos 4 \beta)
$$

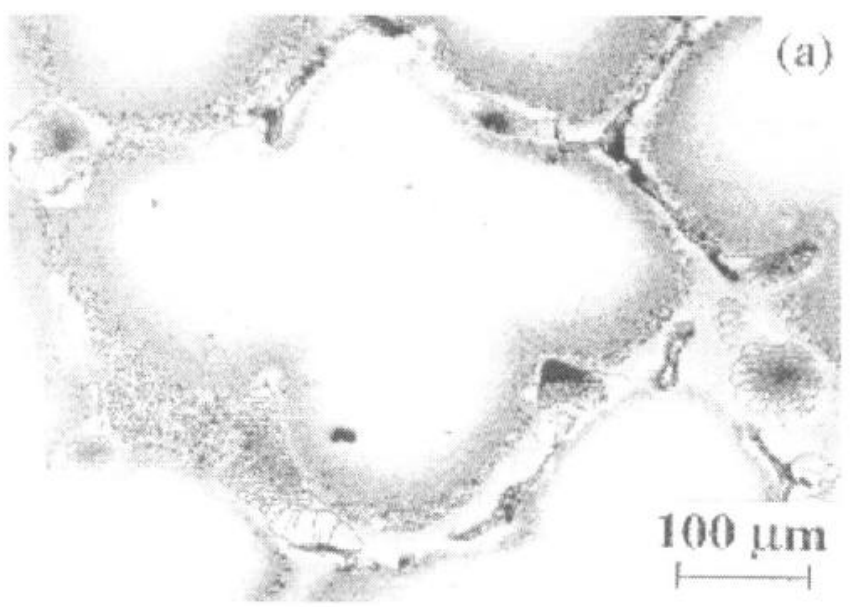

Fig. 1: Cross-section through a CMSX-4 dendritic array obtained by directional solidification.

The amplitude factor $A$ determines the amount of anisotropy of the shape function. For $A=0$ a circular shape is observed corresponding to cellular growth, the maximum value is $A=1$. The unit cell is depicted in Fig. 2.

The function is chosen according to the description of the surface free energy of fcc crystals ${ }^{14}$. Under the conditions of high thermal gradients and low cooling rate no tertiary arms are observed and the shape function is a good geometric approach.

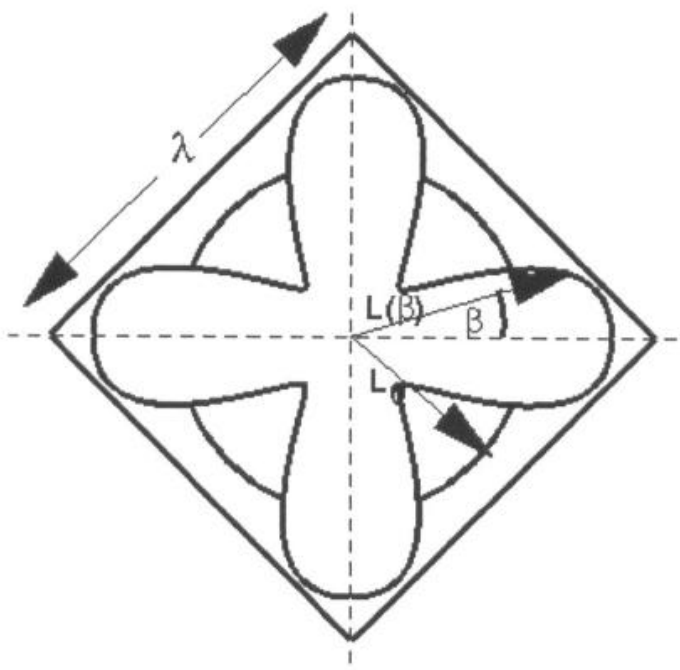

Fig. 2: Dendritic unit cell used for the $S X-$ and the SCPF-Model.

The side length of the unit cell is correlated to the dendritic primary spacing $\lambda_{1}$ and can be estimated from the thermal gradient $\mathrm{G}$ and the solidification velocity $v$ using the theory of Hunt ${ }^{15}$ :

$$
\lambda_{1}=K G^{-1 / 2} v^{-1 / 4}
$$

According to Goldschmidt ${ }^{16}$ the factor $K$ can be considered as a unique constant for superalloys. Alternatively $K$ can be estimated from experiments.

\section{SX-Model: Scheil approximation for SX-alloys}

Within the SX-Model back diffusion is neglected as no fast diffusing interstitial elements are present in single crystal alloys and diffusion in the liquid is considered to be complete (Scheil approximation). So the solid fraction $f_{s}$ is only a function of temperature $T$ which itself depends on solidification time $t$ :

$$
\begin{aligned}
& f_{s}=f(T) \\
& T=T_{0}-G v t
\end{aligned}
$$

In Eq. $4 G$ denotes the thermal gradient and $v$ the solidification velocity along this gradient.

Within the unit cell model $f_{\mathrm{s}}$ corresponds to the quotient of the dendrite area and the total area of the unit cell:

$$
f_{s}=\frac{F}{\lambda_{1}^{2}}=\frac{1}{\lambda_{1}^{2}} \int 0.5 L^{2}(\beta) d \beta
$$

At later solidification times growth of the dendrite is hindered by neighboring dendrites. In this case the position of the solidliquid interface may exceed the maximum length given by the primary dendrite arm spacing. Then the size of the shape function must be corrected properly ${ }^{7}$ to get the correct value for $f_{\mathrm{s}}$. 
The amplitude factor $A$ can be estimated using an empirical relation proposed by $\mathrm{Ma}^{7}$. The amplitude factor depends on the solidification velocity $v$ and on the critical value $v_{\mathrm{e}}$, at which the solidification front shows fully developed cells:

$$
A=1-\left[\frac{v_{c}}{v}\right]^{\frac{1}{2}}
$$

In case of $v$ being equal to $v_{c} A$ equals to zero corresponding to a circular dendritic cross section. At $\nu=y_{\mathrm{c}}$ the planar solidification front shows first instabilities, at a higher value $v_{t}$ the transition from cellular to dendritic growth is observed. $v_{\mathrm{c}}$ is taken to lic halfway between $v_{c}$ and $v_{1}$ (Fig. 3).

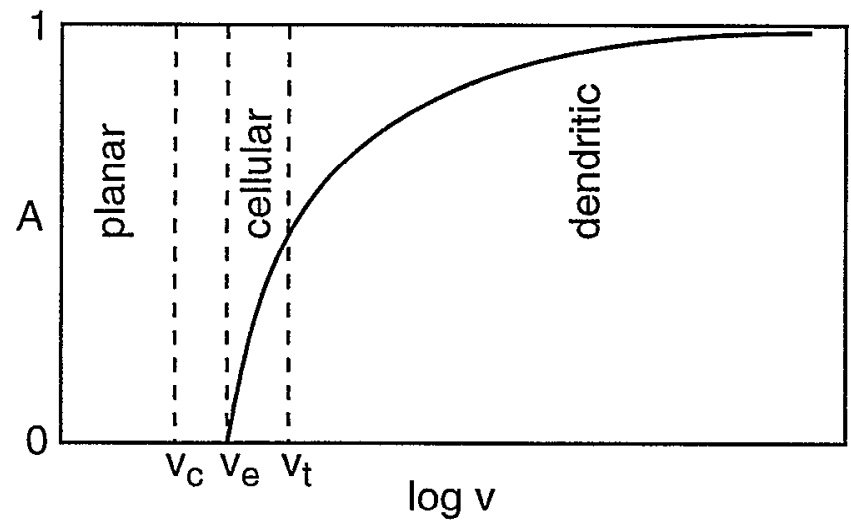

Fig. 3: Relation between the amplitude factor $A$ and the solidification velocity $v$

To model the evolution of the shape function with time, the temporal evolution of the fraction solid $f_{s}$ is needed. For SXsuperalloys back diffusion can be neglected and the multicomponent Scheil model is applied. It is implemented using a FORTRAN code, that calls subroutines of the TQ-interface provided by the thermodynamic software Thermo-Calc ${ }^{12}$. The thermodynamic description for the multicomponent CMSX-4 was developed by Thermotech Ltd. and Rolls-Royce plc. was used $^{13}$. Further details about the model can be taken from elsewhere $^{7.17}$.

\section{The Shape-Constrained Phasefield Model}

For conventional superalloys the Scheil model is not suitable for prediction of microsegregation and secondary phase precipitation because of fast back diffusion of the interstitial elements. Therefore the more refined numerical Shape-Constrained Phasefield (SCPF-) Model is used.

\section{Implementation of the shape function on a numerical finite differences grid}

Although this model like the SX-Model is based on the unit cell approach (Fig. 2), the growing shape function is treated in a numerical manner. The shape function is projected onto a finite difference grid (Fig. 4). Like in phasefield methods a diffuse interface is applied to avoid the necessity of front tracking and to allow the application of a single diffusion algorithm in the whole multiphase region. A phasefield parameter $\phi_{\alpha}$ is defined, which denotes the phase fraction of all phases $\alpha$. The diffuse interface region is created by application of a hyperbolic tangent function perpendicular to the interface (Fig. 4). The use of isolating boundary conditions at the border of the unit cell ensures proper treatment of the interactions with neighboring dendrites for a regular dendritic array. For reasons of symmetry only a quarter of the unit cell needs to be calculated.

\section{a) Projection onto the grid}
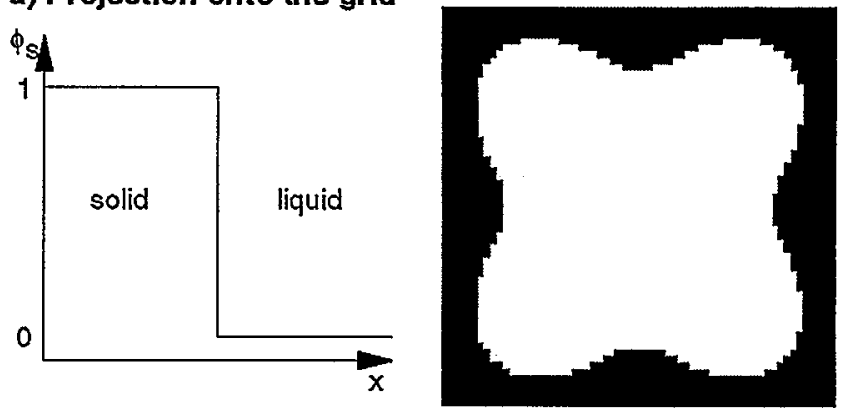

\section{b) Smoothening with a}

$$
\text { tanh-Profile }
$$
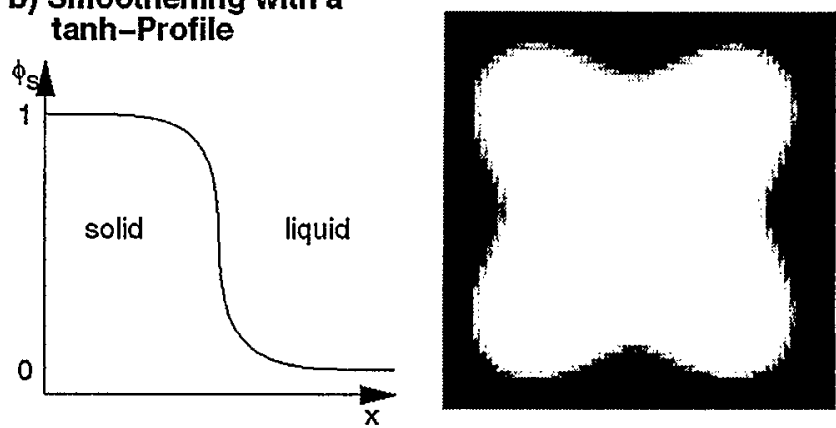

Fig. 4: Implementation of the unit cell in the shape-constrained phasefield model on a finite differences grid with diffuse interface.

\section{Multicomponent Diffusion}

The diffusion algorithm used in the SCPF-Model is based on the Multi-Phasefield diffusion concept ${ }^{18}$ and has been generalized for multicomponent systems. The total flux of component $\mathrm{k}$ $\mathrm{dc}^{k} / \mathrm{dt}$ in the multiphase region is considered as the sum of the fluxes in the individual phases weighted by their phasefield parameter $\phi_{\alpha}$, which corresponds to the local volume fraction of phase $\alpha$ (volume average approach):

$$
d c^{k} / d t=\nabla \sum_{\alpha=1}^{N} \phi_{\alpha} D_{\alpha}^{k} \nabla c_{\alpha}^{k}
$$


The diffusion coefficients $D_{\alpha}{ }^{k}$ in all phases $\alpha$ are regarded as constant, but could also easily be implemented as temperaturedependent. The composition of phase $\alpha$ is obtained from the partition coefficients $K_{\beta \alpha}$ between phase $\alpha$ and all other phases $\beta$ and the phasefield parameter $\phi_{\beta}$ :

$$
c_{\alpha}^{k}=\frac{c^{k}}{\sum_{\beta=1}^{N} \phi_{\beta} K_{\beta \alpha}^{k}}
$$

Thermodynamic coupling

For $1 \mathrm{~N} 706$ a commercial $\mathrm{Ni}$ database from Thermotech Ltd. with 11 elements was used and from there the partition coefficients and the undercooling of the interfaces are calculated.

As in the SX-Model thermodynamic coupling is done using the TQ-interface of the software Thermo-Calc. Partition coefficients must be evaluated for all interface cells to obtain the phase compositions needed for the diffusion algorithm (Eq. 8).

The growth kinetics of the shape function is determined using the average solutal undercooling $\Delta T_{\text {solutal }}$ of the interface, calculated from the average composition by Thermo-Calc subroutines. Additionally the curvature contribution $\Delta T_{\text {curvature }}$ is calculated to obtain the kinetic undercooling of the interface:

$$
\Delta T=\Delta T_{\text {xalual }}+\Delta T_{\text {curvature }}
$$

An exponential kinetic equation is used which is built up as a sum two terms for forward and backward motion of the solidification front:

$$
v=v_{0}\left[\exp \left(\frac{\Delta T}{\Delta T_{0}}\right)-\exp \left(\frac{-\Delta T}{\Delta T_{0}}\right)\right]
$$

Here $v_{0}$ and $\Delta T_{0}$ are numeric parameters which describe the mobility of the interface.

Separately the undercooling of the region of the secondary arm is calculated to obtain the growth velocity in the [001] direction of the dendrite:

$$
v_{(\mathrm{oON})}=v_{0} \kappa\left[\exp \left(\frac{\left.\Delta T_{(\mathrm{(x)})}\right)}{\Delta T_{0}}\right)-\exp \left(\frac{-\Delta T_{(001)}}{\Delta T_{0}}\right)\right]
$$

$\kappa$ is the kinetic anisotropy factor which raises the mobility of the [001] direction. It is assumed to be 1.05 in the calculations below. With the two velocities $v$ and $v_{[001]} L_{0}$ and $L\left(0^{\circ}\right)$ in Eq. 1 can be obtained and the amplitude factor $A$ can be calculated for eich time step:

$$
A(t)=\frac{L\left(0^{\circ}, t\right)}{L_{0}(t)}-1
$$

By this way $A(t)$ is included as additional variable to allow the system to change from cellular to dendritic growth depending on the growth conditions, the diffusion coefficients and surface energy.

\section{Precipitation of interdendritic phases}

Precipitation of secondary phases is an important factor in solidification processes, as they can be detrimental for the mechanical properties or can raise the homogenization time ncccssary for thcir rcmoval. In this model secondary phases are included as circular shape functions, i.e. the amplitude factor $\mathrm{A}$ is restricted to zero. Nevertheless after complete solidification the precipitates are not necessarily circular because they can be partially overgrown by the primary dendrites. Thus the exact shape of the precipitates cannot be predicted by the model, but the volume fraction and the spatial distribution in the unit cell. Interactions between solid phases are not included.

Nucleation is assumed to take place at the solid-liquid interface of the primary dendrite. For nucleation a certain undercooling $\Delta T_{\text {nuc }}$ is necessary. The local undercooling is calculated using Thermo-Calc and the thermodynamic database.

\section{Experimental Details}

For validation of the models two samples of CMSX-4 and INCONEL 706 were directionally solidified in a Bridgman furnace. The nominal compositions of the two alloys are shown in Tab. 1 and 2. The CMSX-4 sample was solidified at a velocity of $1.0 \mathrm{~mm} / \mathrm{min}$ with a thermal gradient of $2.85 \mathrm{~K} / \mathrm{mm}$, the IN706 sample at $\nu=2 \mathrm{~mm} / \mathrm{min}$ and $G=15 \mathrm{~K} / \mathrm{mm}$.

Tab. 1: Nominal and measured compositions of CMSX-4

\begin{tabular}{|c|c|c|c|c|c|c|c|c|c|c|}
\hline & $\mathrm{Ni}$ & $\mathrm{Co}$ & $\mathrm{Cr}$ & $\mathrm{Ta}$ & $\mathrm{W}$ & $\mathrm{Al}$ & $\mathrm{Re}$ & $\pi$ & $\mathrm{Mo}$ & $\mathrm{Hf}$ \\
\hline nom. & bal. & 9.0 & 6.5 & 6.5 & 6.0 & 5.6 & 3.0 & 1.0 & 0.3 & 0.1 \\
\hline EDX & bal. & 10.02 & 6.32 & 6.04 & 5.83 & 5.0 & 2.78 & 1.0 & 0.36 & 0.51 \\
\hline
\end{tabular}

Tab. 2: Nominal and measured compositions of IN706

\begin{tabular}{|c|c|c|c|c|c|c|c|c|c|c|}
\hline & $\mathrm{NI}$ & $\mathrm{Fe}$ & $\mathrm{Gr}$ & $\mathrm{Nb}$ & $T I$ & $A l$ & $M g$ & $S I$ & $C$ & $B$ \\
\hline nom. & bal. & 37.4 & 16.0 & 2.9 & 1.75 & 0.2 & 0.1 & 0.1 & 0.02 & 0.004 \\
\hline EDX & bal. & 38.0 & 16.9 & 3.0 & 1.65 & - & - & - & 0.01 & - \\
\hline
\end{tabular}

Composition measurements of IN706 were made using energy dispersive X-ray analysis (EDX, Type Oxford Link-Isis). For CMSX-4 microprobe measurements (WDX, Type Cameca SX 50) were necessary to distinguish between $\mathrm{W}, \mathrm{Re}$ and Ta. For 'each alloy one linescan along the [001]-axis (secondary arm direction) and one in the [011]-direction was chosen. The exact positions are indicated in Fig. 5 and 6. 


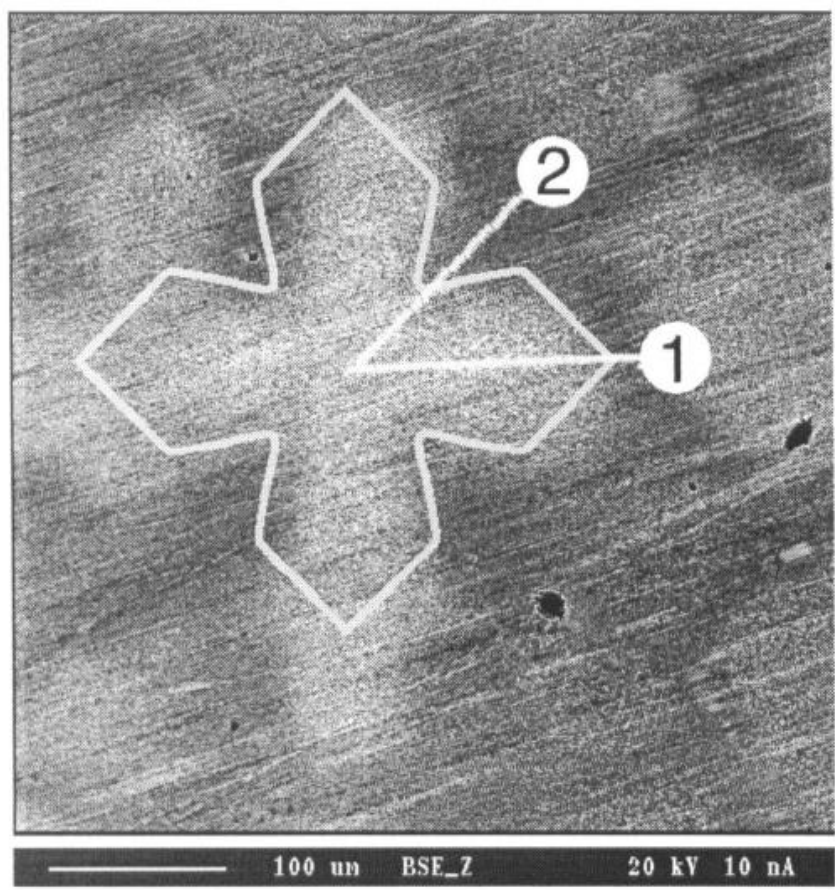

Fig. 5: Scan paths on the CMSX-4 sample. Scan 1 goes from the center along the [001]-axis, scan 2 crosses the region between the secondary arms.

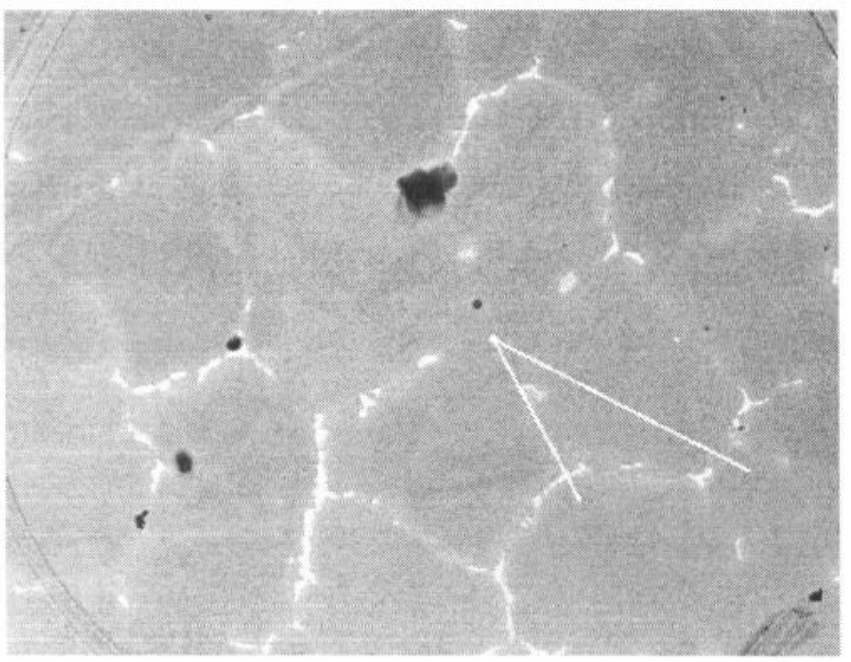

Fig. 6: Scan paths on the IN706 sample. Scan 1 goes from the center along the [001]-axis, scan 2 crosses the region between the secondary arms.

\section{$\underline{\text { Experimental and Numerical Results }}$}

\section{$\underline{\text { SX-Model }}$}

For the SX-Model the critical velocity $v_{\mathrm{e}}$ for the cellular-dendritic transition (Fig. 3) must be obtained from additional experimental data with very low solidification velocities. This was done for CMSX-4, where beginning front instabilities were found at $v_{\mathrm{c}}=0.009 \mathrm{~mm} / \mathrm{min}$. Fully developed cells were formed at $v_{\mathrm{e}}=0.0193 \mathrm{~mm} / \mathrm{min}$. From these values the amplitude factor $A$ can be estimated to 0.87 .

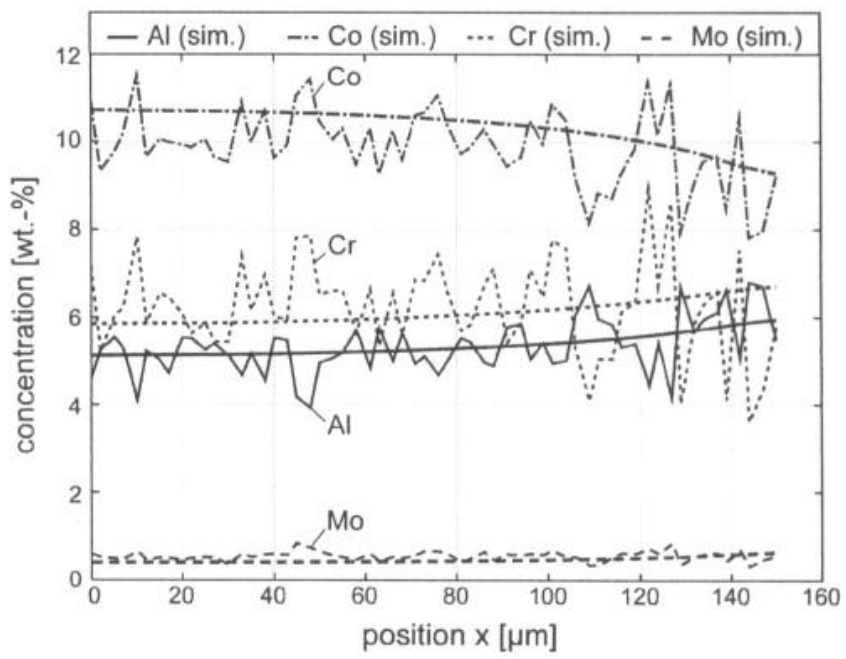

Fig. 7: Comparison between composition profiles of $\mathrm{Al}$, $\mathrm{Co}, \mathrm{Cr}$ and Mo obtained from scan 1 (Fig. 5) and those calculated using the SX-Model.

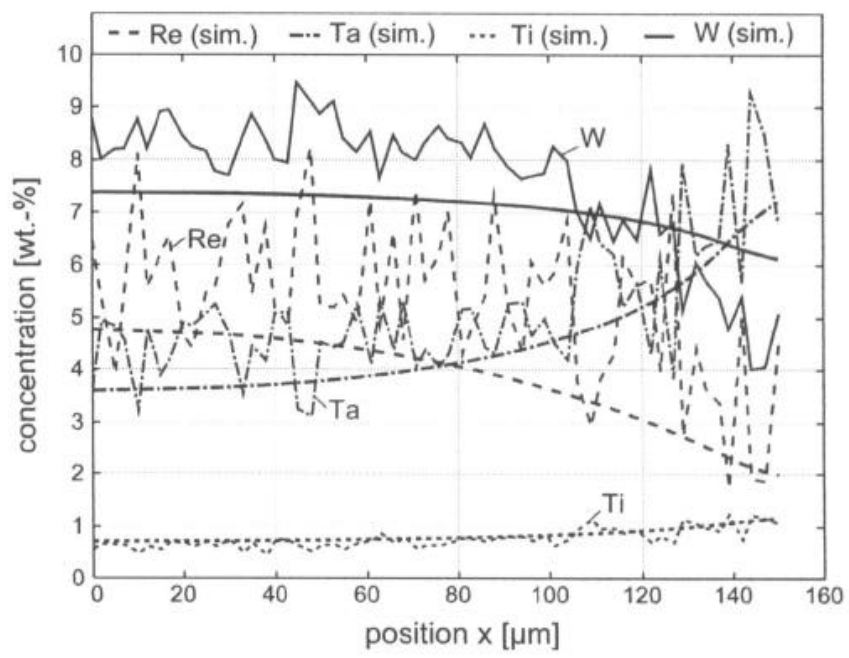

Fig. 8: Comparison between composition profiles of $\mathrm{Ti}$, W, Re and Ta obtained from scan 1 (Fig. 5) and those calculated using the SX-Model. 


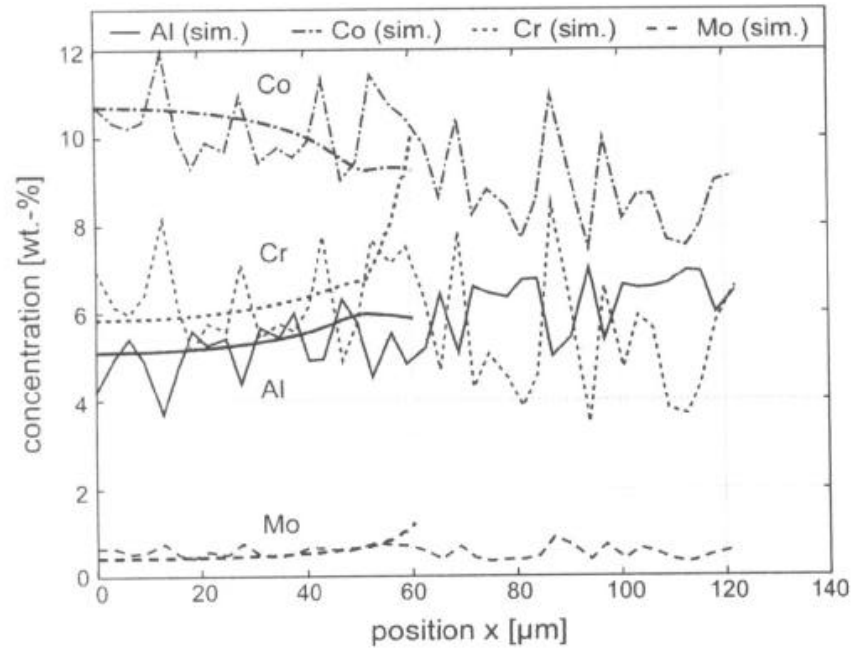

Fig. 9: Comparison between composition profiles of $\mathrm{Al}$, Co, $\mathrm{Cr}$ and Mo obtained from scan 2 (Fig. 5) and those calculated using the SX-Model.

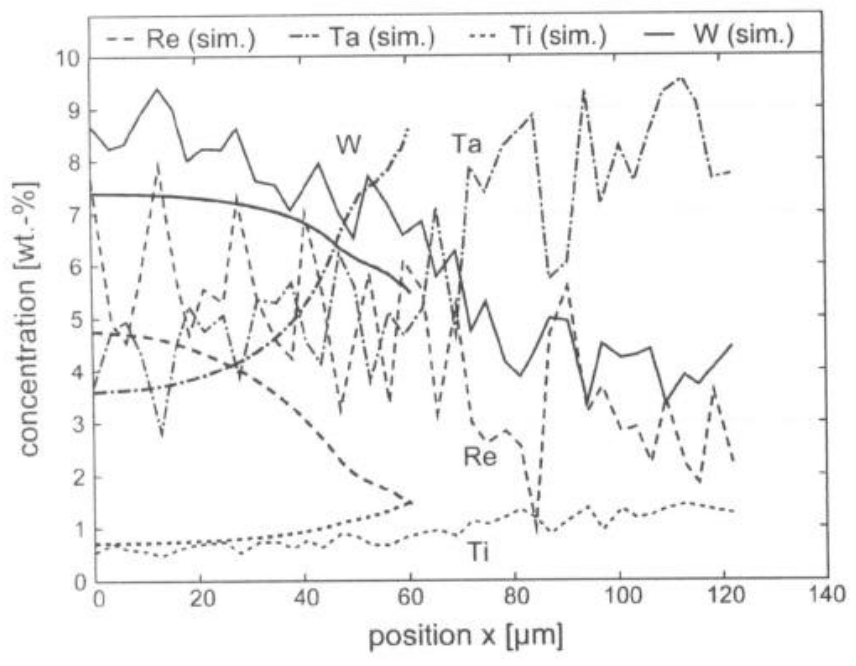

Fig. 10: Comparison between composition profiles of $\mathrm{Ti}$, W, Re and Ta obtained from scan 2 (Fig. 5) and those calculated using the SX-Model.

For simulation a thermodynamic database developed by Thermotech Ltd. and Rolls-Royce plc. has been used. This database has been made available by ABB company, which holds exclusive rights on this data. All elements which are present in CMSX-4 were used in the simulation with exception of $\mathrm{Hf}$.

The comparison between simulation and experimental results is shown in Fig. 7-10. The large oscillations of the microprobe curves are due to secondary $\gamma^{\prime}$ particles of about the same size as that of the electron beam used for measurements. The calculated curves match fairly well to the experimental composition profiles for $\mathrm{Al}, \mathrm{Co}, \mathrm{Cr}$ and $\mathrm{Mo}$ in Fig. 7 and for $\mathrm{Ti}$ and $\mathrm{Ta}$ in
Fig. 8 (Scan 1), while the values are too low for $\mathrm{W}$ and $\mathrm{Re}$. The same tendency is observed for the [011] direction (scan 2).

\section{Shape-constrained phasefield model}

For calculation of IN706 solidification the Ni-Data from Thermo-Tech covering the elements $\mathrm{Ni}-\mathrm{Al}-\mathrm{Co}-\mathrm{Cr}-\mathrm{Fe}-\mathrm{Mo}-$ $\mathrm{Nb}-\mathrm{Ti}-\mathrm{Zr}-\mathrm{B}-\mathrm{C}$ was used. To reduce computation time the system was reduced to $\mathrm{Ni}-\mathrm{Cr}-\mathrm{Fe}-\mathrm{Nb}-\mathrm{Ti}-\mathrm{C}$. The diffusion coefficients of all elements were set to $1.010^{-9} \mathrm{~m}^{2} / \mathrm{s}$ in the liquid and to $1.010^{-12} \mathrm{~m}^{2} / \mathrm{s}$ in all solid phases with the exception of carbon in $\gamma^{\prime}$ and MC carbides. For these phases the diffusivity was chosen to be $1.0^{-10^{-10}} \mathrm{~m}^{2} / \mathrm{s}$.

Nucleation was allowed for MC carbides, Laves and $\eta$ phase using a critical undercooling of $5 \mathrm{~K}$ for carbides and $2 \mathrm{~K}$ for the other phases. Under the applied conditions only carbides and Laves phase occurred in simulation while a micrograph of the interdendritic region shows also some $\eta$ needles at the border of the Laves phase (Fig. 11).

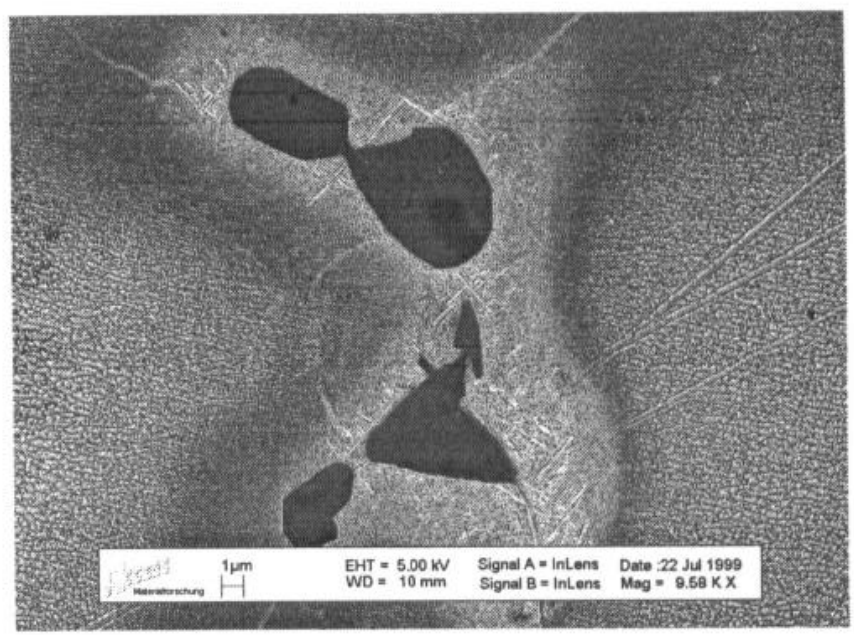

Fig. 11: Micrograph of the interdendritic region of a IN706 sample showing Laves phase and $\eta$ needles.

Fig. 12 shows the calculated microstructure and composition distribution for $\mathrm{C}, \mathrm{Cr}, \mathrm{Fe}, \mathrm{Nb}$ and $\mathrm{Ti}$ for a simulation which corresponds to the experimental conditions of the IN706 solidification experiments $\left(\mathrm{d} T / \mathrm{dt}=-0.5 \mathrm{~K} / \mathrm{s}, \lambda_{1}=200 \mu \mathrm{m}\right)$. Due to their different chemical compositions carbides and Laves phase which formed at the end of solidification in the remaining interdendritic melt can easily be distinguished from the primary dendrite. In this simulation $\mathrm{MC}$-carbides show the tendency to form diffuse patterns as they grow at the same velocity as the primary dendrite. This could be a hint for eutectic growth conditions.

In Fig. 13-16 the EDX composition profiles of the IN706 sample (Fig. 6) are compared to the calculation in Fig. 12. Although there is a qualitative agreement remarkable differences can be 

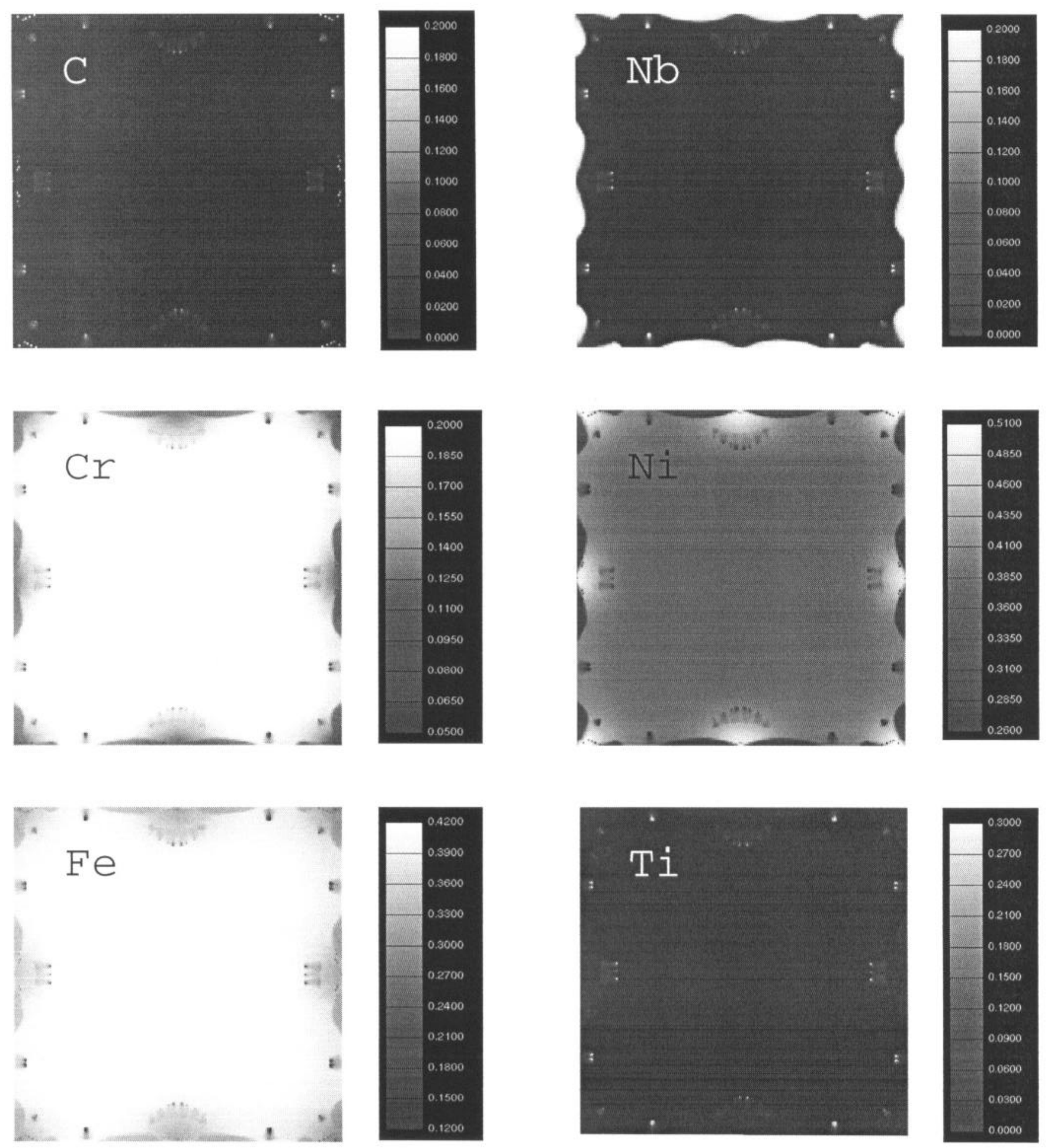

Fig. 12: Composition distribution of $\mathrm{C}, \mathrm{Cr}, \mathrm{Fe}, \mathrm{Nb}, \mathrm{Ni}$ and $\mathrm{Ti}$ in weight fractions calculated using from the SCPF-Model 
seen between measured and calculated profiles. This is primarily caused by the position of precipitates, which heavily influence the composition due to their strong segregation. While the experimental Scan 1 does not cross any precipitate (Fig. 6) carbides and Laves phase are formed in this line in simulation. On the contrary Scan 2 shows two precipitation regions.

With EDX no carbides were found at all on the IN706 sample. The assumption for simulation, that carbides form at the $\gamma$-liquid interface with a nucleation undercooling of $5 \mathrm{~K}$ is very arbitrary, but affects strongly the simulation results.

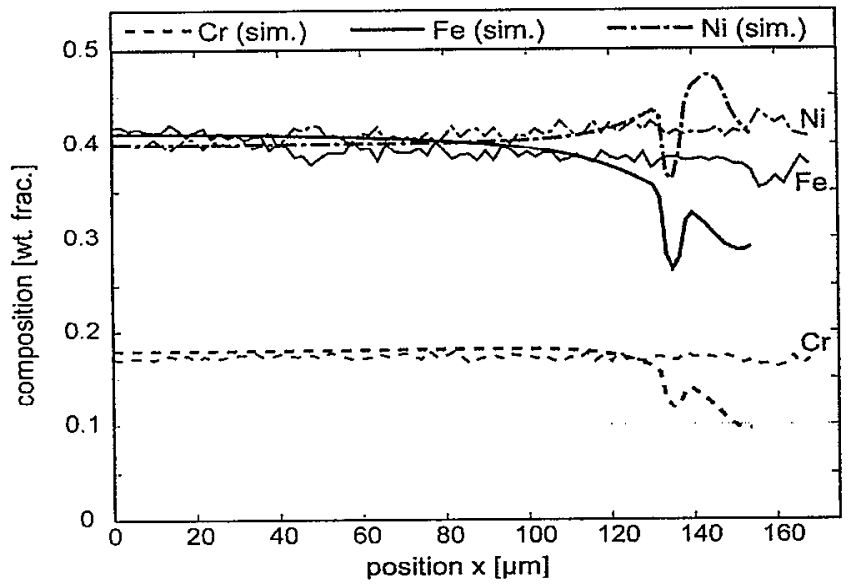

Fig. 13: Comparison between composition profiles of $\mathrm{Ni}$, Fe and Cr obtained from scan 1 (Fig. 6) and those calculated using the SCPF-Model.

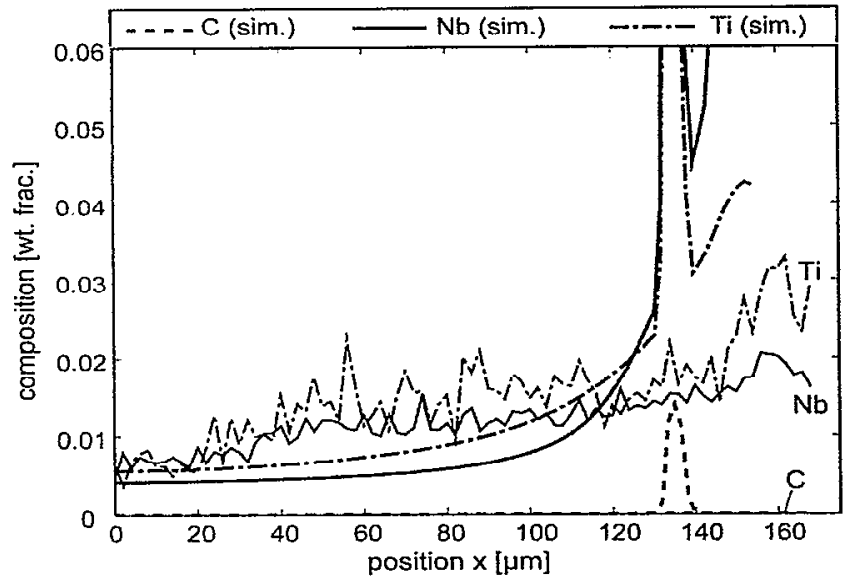

Fig. 14: Comparison between composition profiles of $\mathrm{Nb}$, and $\mathrm{Ti}$ obtained from scan 1 (Fig. 6) and those calculated using the SCPF-Model.

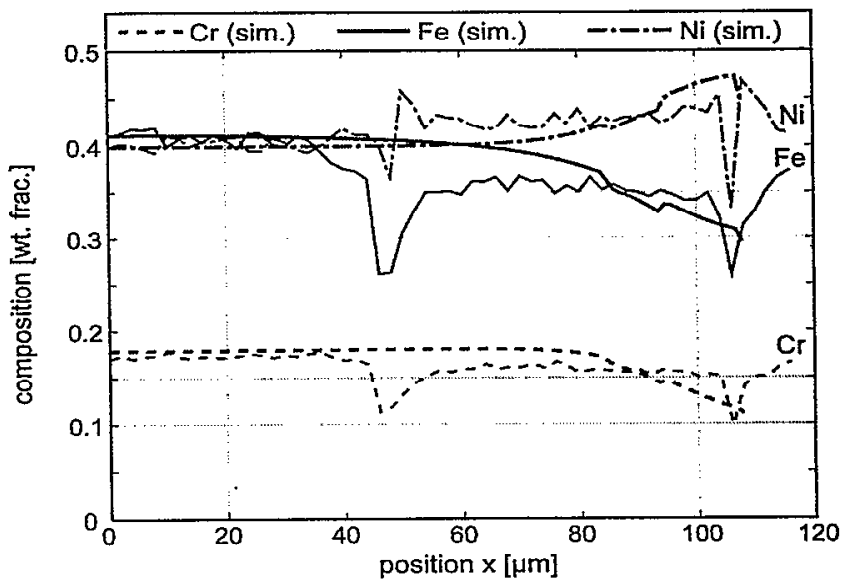

Fig. 15: Comparison between composition profiles of $\mathrm{Ni}$, $\mathrm{Fe}$ and $\mathrm{Cr}$ obtained from scan 2 (Fig. 6) and those calculated using the SCPF-Model.

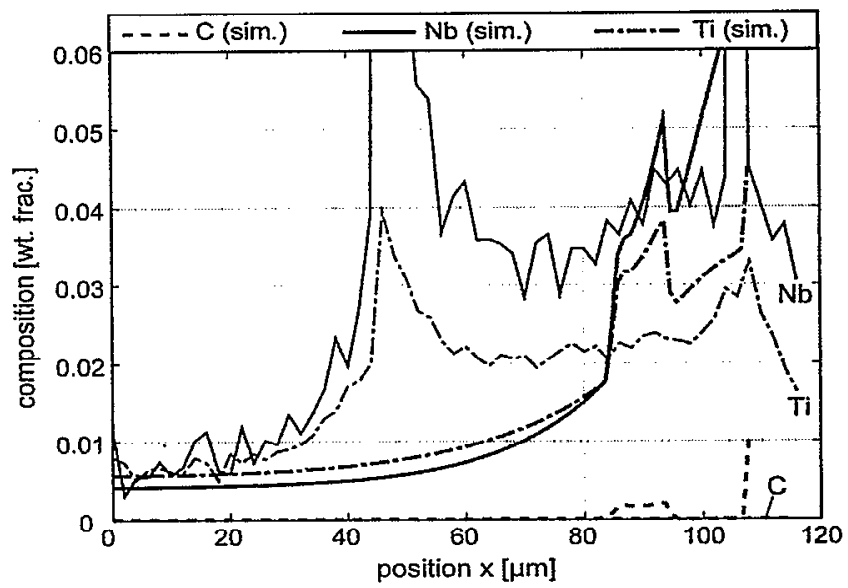

Fig. 16: Comparison between composition profiles of $\mathrm{Nb}$ and $\mathrm{Ti}$ obtained from scan 2 (Fig. 6) and those calculated using the SCPF-Model.

In the experimental Scan 1 all elements except $\mathrm{Cr}$ show an unsteady behavior at about $40 \mu \mathrm{m}$ which could be due to the formation of precipitates in the lower interdendritic region. Also at the edge of the dendrite the composition gradients generally seem to be overestimated. Such effects can be due to axial diffusion in the interdendritic melt and are not included in the two-dimensional SCPF-model. Another explanation could be a certain homogenization during the slow cooling process after complete solidification, which has not becn taken into account in simulation. 


\section{$\underline{\text { Discussion }}$}

The agreement between the results of the SX-Model and the WDX profiles obtained from the CMSX-4 sample is quite good taking into account the oscillations in the WDX signal due to secondary $\gamma^{\prime}$ particles. This is ensured by using thermodynamic data which are based on a huge number of experiments and by incorporating a reasonable shape function for the dendrite into the model. The model is restricted to systems where multicomponent Scheil conditions hold, and formation of interdendritic precipitates is not included.

For systems like IN706, where precipitates of very different composition compared to the primary dendrite can heavily influence the microsegregation profiles and where interstitial elements exhibit considerable back diffusion the more refined Shape-Constrained Phasefield (SCPF-)Model is more adequate.

Due to the complexity of the system no perfect match of simulation and experiment should be expected. Too many factors like diffusion coefficients and nucleation undercoolings are unknown. Nevertheless a principially good agreement with the experimental data can be seen in Fig. 13-16 keeping in mind the strong effect of the precipitation position which are different in simulation and experiment and the fact that no carbides were found at all in the micrographs. They could have formed in the deeper interdendritic melt and reduced the carbon content in the measured cross section through axial diffusion. Other reasons for the deviations are unknown parameters, which have a strong influence on the results of the simulation. These are physical variables like diffusion coefficients, which are only estimated due to the lack of experimental data, the critical nucleation undercooling of the different phases or the interfacial energies, which determine the curvature undercooling. These parameters in future could partially be obtained by fitting the calculation results to the experiment.

\section{Outlook}

For validation of the SCPF-Model further experiments at different conditions and with different alloys will be done. Of specinl importance with IN706 is the role of carbides which will be studied with carbon-enriched samples. On this basis more reasonable nucleation conditions in simulation should lead to a better agreement between simulation and experiments.

Compared to the Phasefied Mode $1^{18,19}$ the use of the shape function concept in the SCPF-Model allows a dramatic reduction of the calculation effort. This is mainly duc to the online coupling of thermodynamic data to obtain the driving force for the growing dendrite, which is considerably reduced by use of shape functions. But as the shape function approach is compatible to the Phasefield algorithm, the latter could be used just for secondary phases while the primary dendrite is further treated as shape function. With this combination homogenization and secondary prccipitation after complete solidification could be included which would greatly enhance the capability of the model for microstructure prediction and alloy design.

\section{Acknowledgment}

The authors wish to thank DFG (Deutsche Forschungsgesellschaft) for financial support and ABB for supplying the database for CMSX-4 simulations.

\section{References}

1. H. D. Browdy and M. D. Flemings, "Solute Redistribution in Dendritic Solidification", Trans. AIME, 236 (1966) 615-624.

2. D. J. Allen, J. D. Hunt, "Diffusion in the Semi-Solid Region During Dendritic Growth", Metall. Trans. A, 10A (1979) 1389-1397.

3. A. Roósz, E. Halder and H. E. Exner, "Numerical Calculation of Microsegregation in Coarsened Dendritic Microstructures", Mater. Sci. Technol., 2 (1986) 1149-1155.

4. T. Kraft, M. Rettenmayr and H. E. Exner, "An Extended Numerical Procedure for Predicting Microstructure and Microsegregation of Multicomponent Alloys", Modelling Simul. Mater. Sci. Eng. 4 (1996) 161-177.

5. T. Himemiya and T. Umeda, "Solute Redistribution Model of Dendritic Solidification Considering Diffusion in Both the Liquid and Solid Phases", ISIJ International, 38 (1998), No. 7, 730-738.

6. D. Ma and P. Sahm, "Microsegregation in Cellular Microstructure", Metall. Trans. A, 23A (1992) 3377-3381.

7. D. Ma, "Modellierung des Dendritenwachstums und der Mikroseigerung bei gerichteter Erstarrung, Teil I: Entwicklung eines analytischen Mikromodells", Gießereiforschung, 1 (1998) $29-34$.

8. T. Kraft and H. E. Exner, "Numerische Simulation der Erstarrung, Teil2: Mikroseigerung in ternären und höherkomponentigen Legierungen", Z. Metallkd. 87 (1996) 652-660.

9. A. J. W. Ogilvy and D. H. Kirkwood, "A Model for the Numerical Computation of Microsegregation in Alloys", Appl. Sci. Res. 44 (1987) 43-49.

10. A. Roósz and H. E. Exner, "Ternary Restricted-Equilibrium Phase Diagrams - I. A First Report: General Principles and Definitions; II. Practical Application: Aluminium-Rich Corner of the Al-Cu-Mg System", Acta metall. mater. 38 (1990) 2003-2008, 2009-2016.

11. N. Saunders and A. Miodownik: CALPHAD, Calculation of Phase Diagrams, A Comprehensive Guide; Elsevier S. Ltd. 1998, ISBN U-08-042-129-6.

12. B. Sundman, B. Jansson and J. O. Anderson, "The ThermoCalc Databank System", CALPHAD, 9 (1985) 153-190.

13. N. Saunders, "Phase Diagram Calculations for Ni-Based Superalloys", Superalloys 1996, (Warrendale, PA: Met. Soc. AIME., 1996), 101-110. 
14. D. A. Kessler, J. Koplik and H. Levine, "Steady-State Dendritic Crystal Growth", Phys. Rev. A, 31 (1986) 3352-3357.

15. J. D. Hunt, Solidification and Casting of Metals, Vol. 192. The Metal Society, London.

16. D. Goldschmidt, "'Einkristalline Gasturbinenschaufeln aus Nickelbasis-Legierungen, Teil I", Mat.-wiss. u. Werkstofftech., 25 (1994) 311-320.

17. U. Grafe et al., "Calculation of Microsegregation for the Directionally Solidified Superalloy CMSX 4 Using a Pseudo 2Dimensional Model", Modelling of Casting, Welding and Advanced Solidification Processes VIII, The Minerals, Metals \& Materials Society, 1998, 227-234.

18. J. Tiaden et al., "The multiphase-field model with an integrated concept for modelling solute diffusion", Physica D, 115 (1997) 73-86.

19. I. Steinbach et al., "A Phase Field Concept for Multiphase Systems", Pliysica D, 94 (1996) 135-147. 